\title{
Improving the secondary prevention of ST-elevation myocardial infarction
}

\author{
Authors: Diarmuid Chevalier, ${ }^{\mathrm{A}}$ Yen Jei Chen, ${ }^{\mathrm{B}}$ Gary O'Reilly, ${ }^{\mathrm{C}}$ Kenneth Wong, ${ }^{\mathrm{D}}$ Billal Patel ${ }^{\mathrm{D}}$ and Alison Seed ${ }^{\mathrm{D}}$
}

\section{Introduction}

Secondary prevention medications following ST-elevation myocardial infarction (STEMI) have been established with NICE and European Society of Cardiology guidelines. ${ }^{1,2}$ In practice, while most patients with STEMI are discharged with the correct secondary prevention medications, ${ }^{3}$ titration advice to primary care physicians can be lacking and the dosage of ACE inhibitor and beta blocker at discharge may be subtherapeutic. ${ }^{4}$

Medication titration is essential post-MI to prevent future cardiac events. Prognostic benefits have largely been shown in studies which used these medications in high doses. ${ }^{4-6}$ As such, NICE ${ }^{1}$ gives a five-point guideline on the information to be included in every discharge summary.

We set up a three Plan, Do, Study, Act (PDSA) cycle quality improvement project in Lancashire Cardiac Centre by using a combination of education and a new unified STEMI discharge letter form.

\section{Materials and methods}

Data was collected from the coronary care unit (CCU) STEMI primary percutaneous coronary intervention (PPCI) list, with the following inclusion criteria:

$>$ Admitted through PPCI pathway

$>$ Confirmed STEMI on discharge

$>$ Admission $<5$ days

$>$ Discharged alive

Discharge summaries of patients within the inclusion criteria were reviewed. For the purpose of this QIP, a correct discharge of STEMI patient following PPCI included the following:

> Beta blocker including dose and dose titration advice

$>$ ACEi/ARB including dose and dose titration advice

$>$ Aldosterone antagonist - where clinically appropriate

$>$ Statin - unless contraindicated

> Dual antiplatelet therapy (DAPT)

Baseline data was collected in 2013 when 42 consecutive patients fitting the inclusion criteria were reviewed. Three separate

Authors: ' $G u y$ 's and St Thomas' Hospital Trust; BBlackpool NHS Foundation Trust; ' 'Liverpool Medical School; DBlackpool NHS Foundation Trust

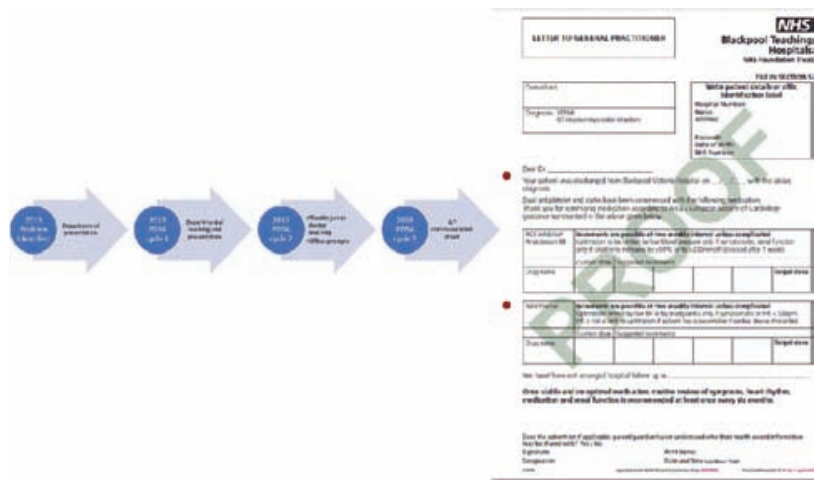

Fig 1. PDSA cycles with ST elevation myocardial infarction (STEMI) discharge GP letter

PDSA cycles (Fig 1) were then implemented between 2017 and 2018. Consecutive patients' notes were reviewed 2 weeks after the introduction of each change. Each cycle was completed only after the previous' data had been analysed and discussed with senior nurses and doctors.

\section{Results and discussions}

\section{PDSA cycle 1 ( $n=33$ )}

Percentage number of discharges with appropriate secondary prevention planning improved from $26 \%$ to $33 \%$ when compared with 2013. However, this was not statistically significant ( $p=0.513)$. PDSA cycle 2 ( $n=26)$

There was a vast improvement to $70 \%$ adherence with national guidelines $(p=0.005)$.

PDSA cycle 3 ( $n=32)$

This general practitioner letter strategy (see Fig 2) achieved a $75 \%$ correct discharge. Compared with PDSA cycle 2, the improvement is statistically insignificant $(p=0.684)$. However, when compared with baseline 2013 data, the improvement is clearly significant ( $p=<0.001)$.

\section{Conclusion}

PDSA cycles 2 and 3 appeared most successful in achieving correctly discharged patients. Compared with the educational 


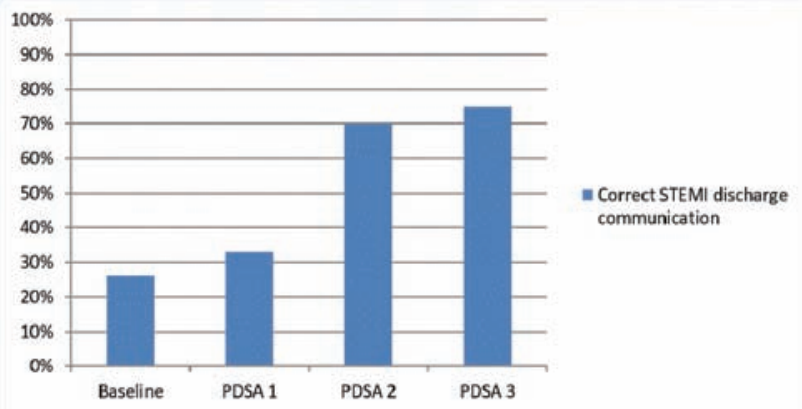

Fig 2. Percentage of patients admitted through the PPCI pathway and with a final diagnosis of ST-elevation myocardial infarction (STEMI) that were correctly discharged, according to NICE guidelines

week for PDSA cycle 2, the STEMI discharge letter was less labour intensive. Furthermore, it is clearer for patients and primary care practitioners. Its introduction requires only a 5 -minute presentation and its supply was maintained by the ward clerk, costing around $£ 30$ for a booklet of 50 . The information is easily reproducible and requires reduced senior input for its maintenance, making it more sustainable as a long-term quality improvement. As such, the STEMI GP letter was unanimously accepted by the cardiology department to be introduced for new teams to use.

\section{References}

1 National Institute for Health and Care Excellence. Myocardial infarction with ST-segment elevation: acute management. Clinical guidance [CG167]. London: NICE, 2013.

2 Ibanez B, James S, Agewall S et al. ESC Guidelines for the management of acute myocardial infarction in patients presenting with ST-segment elevation: The Task Force for the management of acute myocardial infarction in patients presenting with ST-segment elevation of the European Society of Cardiology (ESC). Eur Heart ] 2017;39:119-77.

3 Myocardial Ischemia National Audit Project. How the NHS cares for patients with heart attacks: annual public report April 2014-March 2015. HQIP, 2017.

4 Stewart K, Woothipoom P, Townend J. Achieving the dose: an audit of discharge medication for the secondary prevention of myocardial infarction. Br J Cardiol 2010;17:142-3.

5 Yusuf S, Sleight P, Pogue ] et al. Effects of an angiotensin-converting enzyme inhibitor, ramipril, on cardiovascular events in high-risk patients. N Engl J Med 2000;342:145-53.

6 Simon T, Mary-Krause M, Funck-Bretano C et al. Bisoprolol doseresponse relationship in patients with congestive heart failure: a subgroup analysis in the cardiac insufficiency bisoprolol stufy (CIBIS II). Eur Heart ] 2003;24:552-9. 